\title{
Lie bialgebra structures on 2-step nilpotent graph algebras
}

\author{
Marco A. Farinati* and Alejandra Patricia Jancsa ${ }^{\dagger}$ \\ September 19, 2018
}

\begin{abstract}
We generalize a result on the Heisenberg Lie algebra that gives restrictions to possible Lie bialgebra cobrackets on 2-step nilpotent algebras with some additional properties. For the class of 2-step nilpotent Lie algebras coming from graphs, we describe these extra properties in a very easy graph-combinatorial way. We exhibit applications for $\mathfrak{f}_{n}$, the free 2-step nilpotent Lie algebra.
\end{abstract}

\section{Introduction}

A Lie bialgebra is a triple $(\mathfrak{g},[-,-], \delta)$ where $(\mathfrak{g},[-,-])$ is a Lie algebra and $\delta: \mathfrak{g} \rightarrow \Lambda^{2} \mathfrak{g}$ is a linear map such that

- $\delta$ satisfies the co - Jacobi identity. In Sweedler type notation, if $\delta(x)=x_{1} \wedge x_{2}$ (sum understood), then co-Jacobi condition reads

$$
\delta\left(x_{1}\right) \wedge x_{2}-x_{1} \wedge \delta\left(x_{2}\right)=0 \in \Lambda^{3} \mathfrak{g}
$$

- $\delta$ satisfies the 1-cocycle condition $\delta[x, y]=[\delta x, y]+[x, \delta y] \in \Lambda^{2} \mathfrak{g}$.

Lie bialgebras first appear as classical limit of quantum objects, studying deformations of Hopf algebras and the Quantum Yang-Baxter equation, presented by Drinfel'd. They also appear in geometry as Poisson Lie structures on Lie group, and in algebraic topology as additional algebraic structure on the space of (free homotopy classes of) curves in oriented surfaces. Lie bialgebra structures with underlying complex simple $x$ Lie algebras were classified by Belavin and Drinfel'd [BD] under a nondegenerated assumption (called factorisable case) and after that a lot of people work on semi simple and reductive case. In [FJ] we studied the situation of a Lie algebra $\mathfrak{l}=\mathfrak{g} \times V$ (where $V$ is an abelian factor) and the problem of determine all possible Lie

\footnotetext{
*Member of CONICET. Partially supported by PIP 11220110100800CO, and UBACYT 20021030100481BA, mfarinat@dm.uba.ar.

†Partially supported by PIP 11220110100800CO and UBACYT 20021030100481BA, pjancsa@dm.uba.ar
} 
bialgebra structures supported by $\mathfrak{l}$ in terms of Lie bialgebra structures on $\mathfrak{g}$, and we obtain in that way non factorisable examples for reductive Lie algebras. Although its importance, the classification problem of Lie bialgebras remain a wide open problem. In the opposite side of simple or semi simple case (e.g. solvable or nilpotent) there almost no results, and in some cases is hopeless for obvious reasons: if the underlying algebra $\mathfrak{g}$ is abelian (say $\operatorname{dim} \mathfrak{g}<\infty$ ), to classify all Lie bialgebra structures on $\mathfrak{g}$ is the same as the classification of all Lie algebra structures on the vector space $\mathfrak{g}^{*}$. Surprisingly, if $\mathfrak{g}=\mathfrak{h}_{2 m+1}$, the $2 \mathrm{~m}+1$-dimensional Heisenberg Lie algebra, all Lie bialgebra structures on $\mathfrak{h}_{2 m+1}$ are known from a long time (see [BS] and [SZ]), but for general nilpotent Lie algebras there is nothing else. The nilpotent case is of special importance because after a result of Etingof and Gelaki (see [EG], we thank Milen Yakimov for pointing out this reference), connected Hopf algebras of finite Gelfand Kirillov dimension are in bijection with nilpotent Lie bialgebras.

The origin of this work is the following observation: if $\mathfrak{n}$ is a 2-step nilpotent Lie algebra with center $\mathfrak{z}$, then one can find a linear complement $W$ so that $\mathfrak{n}=\mathfrak{z} \oplus W$. Once we use this (vector space) decomposition, one can see analogies both in our previous work $(\mathfrak{l}=\mathfrak{g} \times V)$ and in the Heisenberg case $\mathfrak{h}_{2 m+1}=W \oplus k z$. Using this point of view we obtain, under mild assumptions, very strong restrictions on all possible co-bracket on a general 2-step nilpotent algebra. Although these restrictions, some nonlinear equations remains to be solved and the general situation is still wide, but restricted to graph algebras the problem is more tractable. Graphs algebras is a very interesting class of 2-step nilpotent algebras; even if in each dimension there are only a finite number of them, they are numerous enough to construct interesting examples and counterexamples in geometry (see [LW] and [GGI]).

The paper is organized as follows: in section 1 we study general properties of 2step nilpotent Lie algebras. The main results are Theorem 1.4, where the Heisenberg case is generalized to arbitrary 2-step algebras, and Theorem 1.8 and its reciprocate 1.9, where the analogy with our previous work [FJ] is used. In section 2 we specialize to graph algebras. The main results of section 2 are Theorems 2.4 and 2.5 where the two algebraic nice hypothesis needed in section 1 are translated in terms of the combinatorics of the graph. Section 3 deals with an application of a special class Lie bialgebras, the ones whose co-bracket annihilates the center (these class largely include the coboundary ones). Inside this class we study $\mathfrak{f}_{n}$, the free 2-step nilpotent algebra on $n$ generators, that in terms of graph corresponds to the complete graph $K_{n}$.

Acknowledgements: We would like to thank Leandro Cagliero for several discussion on 2-step nilpotent algebras and his help on clarifying equations at the begining of the project. We would also like to thank Moira Chas and Dennis Sullivan for a generous invitation to Stony Brook where we finish the second part of this work.

\section{Two-step nilpotent Lie bialgebras}

A lie algebra $\mathfrak{n}$ is called 2-step nilpotent if $[\mathfrak{n},[\mathfrak{n}, \mathfrak{n}]]=0$, or equivalently if $[n, \mathfrak{n}] \subseteq \mathfrak{z}$, where $\mathfrak{z}$ is the center of $\mathfrak{n}$. As vector space, the center admits a linear complement; we choose one and call it $W$, so that $\mathfrak{n}=W \oplus \mathfrak{z}$ (as linear vector spaces). We will study Lie cobrackets $\delta$ defined on the underlying Lie algebra $\mathfrak{n}$. If $(\mathfrak{n}, \delta)$ is a Lie bialgebra 
structure, $\delta: \mathfrak{n} \rightarrow \Lambda^{2} \mathfrak{n}$, hence it admits a decomposition via

$$
\mathfrak{n}=W \oplus \mathfrak{z} \rightarrow \Lambda^{2}(W \oplus \mathfrak{z})=\Lambda^{2} W \oplus W \wedge \mathfrak{z} \oplus \Lambda^{2} \mathfrak{z}
$$

So, we may split $\delta$ in several components. In particular, define $\delta_{1}:=\left.\delta\right|_{W} ^{\Lambda^{2} W}$, that is, for $v \in W, \delta_{1}(v)$ is the $\Lambda^{2} W$ - component of $\delta(v)$.

A very important and classical example is the Heisenberg Lie algebra $\mathfrak{n}=\mathfrak{h}_{2 n+1}=$ $W \oplus k z$ where $W=k^{2 n}$ with symplectic form $\omega$ and bracket given by

$$
[v+\lambda z, w+\mu z]=\omega(v, w) z
$$

For the Heisenberg Lie algebra, all possible cobrackets are well-known

Theorem 1.1. ([SZ] and [BS]]) If $\delta(z)=v_{0} \wedge z$ then

$$
\begin{array}{cc}
\delta(v)=\frac{1}{4}\left(\omega\left(v_{0}, v\right) \widehat{\omega}+v_{0} \wedge v\right) & \in \Lambda^{2} W \\
+D(v) \wedge z & \in W \wedge z
\end{array}
$$

where $D$ is a coderivation w.r.t. $\delta_{1}$.

In particular, $\delta_{1}$ is determined by $\delta(z)$, and if $\delta(\mathfrak{z})=0$ then $\delta(v)=D(v) \wedge z \forall v \in W$. We will generalize one direction of the above theorem. We begin with a simple remark

Remark 1.2. If $\delta: \mathfrak{n} \rightarrow \Lambda^{2} \mathfrak{n}$ is a 1-cocycle then $\delta(\mathfrak{z}) \subset\left(\Lambda^{2} \mathfrak{n}\right)^{\mathfrak{n}}$.

Proof. For $x \in \mathfrak{n}, z \in \mathfrak{z}, \operatorname{ad}_{z} \delta x=\left[z, x_{1}\right] \wedge x_{2}+x_{1} \wedge\left[z, x_{2}\right]=0$, hence

$$
0=\delta(0)=\delta[x, z]=\operatorname{ad}_{x} \delta z-\operatorname{ad}_{z} \delta x=\operatorname{ad}_{x} \delta z+0
$$

That is, $\delta z$ is ad $\operatorname{ad}_{x}$-invariant for any $x \in \mathfrak{n}, z \in \mathfrak{z}$.

Corollary 1.3. If $\left(\Lambda^{2} \mathfrak{n}\right)^{\mathfrak{n}}=\Lambda^{2} \mathfrak{z}$ then $\delta(\mathfrak{z}) \subset \Lambda^{2} \mathfrak{z}$.

Even if the Heisenberg Lie algebra does not satisfy $\left(\Lambda^{2} \mathfrak{n}\right)^{\mathfrak{n}}=\Lambda^{2} \mathfrak{z}$, several interesting 2 -step nilpotent Lie algebras do, as we sill show in next section. Now the important simplification will be given by next theorem. We need to introduce some notation.

For $v, w \in W,[v, w] \in \mathfrak{z}$, fix a basis $\left\{z_{i}\right\}_{i=1}^{\operatorname{dim} \mathfrak{z}}$, and write

$$
[v, w]=\sum_{i} T_{i}(v)(w) z_{i}
$$

where $T_{i}: W \rightarrow W^{*}$ is determined by the Lie bracket.

Denote by $[,]^{*}: W^{*} \times W^{*} \rightarrow W^{*}$ the dual bracket, i.e. if $\delta_{1}(v)=v_{1} \wedge v_{2}$ then $[\lambda, \mu]^{*}(v)=\lambda\left(v_{1}\right) \mu\left(v_{2}\right)$. If $\left\{\lambda_{j}\right\}_{j=1}^{\operatorname{dim} W}$ is a basis of $W^{*}$, define $S_{j}: W^{*} \rightarrow W \cong W^{* *}$ so that

$$
[\lambda, \mu]^{*}=\sum_{j} S_{j}(\mu)(\lambda) \lambda_{j}
$$

Theorem 1.4. Let $(\mathfrak{n}, \delta)$ be a 2-step nilpotent Lie bialgebra, then

- $\delta_{1}$ is a Lie coalgebra structure on $W$. 
- Consider $\left\{\lambda_{j}\right\}_{j=1}^{\operatorname{dim} W},\left\{\mathfrak{z}_{i}\right\}_{i=1}^{\operatorname{dim} \mathfrak{z}}$ basis of $W^{*}$ and $\mathfrak{z}$, resp. and $T_{i}$ and $S_{j}$ as before. If in addition $\delta(\mathfrak{z}) \subset \Lambda^{2} \mathfrak{z}$, then

$$
T_{i} S_{j} T_{k}+T_{k} S_{j} T_{i}=0 \forall i, j, k
$$

For instance, in the Heisenberg Lie algebra there is only on $T$, corresponding to the symplectic form $\omega$, which is non degenerate, so the equation $T S_{j} T=0 \forall j$ clearly implies $\delta_{1}=0$.

Proof. We need to check co-Jacobi for $\delta_{1}$. If $z \in \mathfrak{z}$ then $\delta(z) \in\left(\Lambda^{2} \mathfrak{n}\right)^{\mathfrak{n}}$ and one can check that we always have

$$
\left(\Lambda^{2} \mathfrak{n}\right)^{\mathfrak{n}} \subseteq W \wedge \mathfrak{z} \oplus \Lambda^{2} \mathfrak{z}
$$

that is, $\delta(z)$ do has zero component in $\Lambda^{2} W$. Now if if $v \in W, \delta(v) \in \Lambda^{2} W \oplus W \wedge \mathfrak{z} \oplus \Lambda^{2} \mathfrak{z}$ and we can write

$$
\delta(v)=\delta_{1}(v) \operatorname{Mod}\left(W \wedge \mathfrak{z}+\Lambda^{2} \mathfrak{z}\right)
$$

and the component of $\delta(v)$ in $\Lambda^{3} W$ can only arise because of $\delta_{1}$ applied to components of $\delta_{1}(v)$, since $\delta$ applied to elements in $z$ give components with at least one " $\mathrm{z}$ ", that is, components in $\Lambda^{2} W \wedge \mathfrak{z}+W \wedge \Lambda^{2} \mathfrak{z}+\Lambda^{3} \mathfrak{z}$.

The second part is the most interesting, and this part is a generalization of the arguments for the Heisenberg case.

Let $u, v \in W$. Since $\mathfrak{n}$ is 2-step nilpotent then $[u, v] \in \mathfrak{z}$, but also because $\delta(\mathfrak{z}) \subseteq$ $\left(\Lambda^{2} \mathfrak{n}\right)^{\mathfrak{n}}$ and we assume $\left(\Lambda^{2} \mathfrak{n}\right)^{\mathfrak{n}}=\Lambda^{2} \mathfrak{z}$ we have $\delta[u, v] \in \Lambda^{2} \mathfrak{z}$. But also

$$
\delta[u, v]=[\delta u, v]+[u, \delta v]
$$

Let us write

$$
\delta u=\delta_{1} u+\sum_{i} D^{i}(u) \wedge z_{i}+\delta_{W}^{\Lambda^{2} \mathfrak{z}} u
$$

(and similarly for $\delta v$ ) where $\delta_{W}^{\Lambda^{2} \mathfrak{z}} u$ is the component in $\Lambda^{2} \mathfrak{z}$ of $\delta u$ and $D^{i}: W \rightarrow W$ are linear maps describing the $W \wedge \mathfrak{z}$-component. Then $\delta[u, v]=$

$$
\begin{aligned}
=\left[\delta_{1} u+\right. & \left.\sum_{i} D^{i}(u) \wedge z_{i}+\delta_{W}^{\Lambda^{2} \mathfrak{z}} u, v\right]+\left[u, \delta_{1} v+\sum_{i} D^{i}(v) \wedge z_{i}+\delta_{W}^{\Lambda^{2} \mathfrak{z}} v\right] \\
= & {\left[\delta_{1} u+\sum_{i} D^{i}(u) \wedge z_{i}, v\right]+\left[u, \delta_{1} v+\sum_{i} D^{i}(v) \wedge z_{i}\right] } \\
= & {\left[\delta_{1} u, v\right]+\sum_{i}\left[D^{i}(u), v\right] \wedge z_{i}+\left[u, \delta_{1} v\right]+\sum_{i}\left[u, D^{i}(v)\right] \wedge z_{i} } \\
= & \underbrace{\left[\delta_{1} u, v\right]+\left[u, \delta_{1} v\right]}_{\in W \wedge \mathfrak{z}}+\sum_{i} \underbrace{\left(\left[D^{i}(u), v\right]+\left[u, D^{i}(v)\right]\right) \wedge z_{i}}_{\in \Lambda^{2} \mathfrak{z}}
\end{aligned}
$$

hence $\left\{\begin{aligned} 0 & =\left[\delta_{1} u, v\right]+\left[u, \delta_{1} v\right] \\ \delta[u, v] & =\sum_{i}\left(\left[D^{i}(u), v\right]+\left[u, D^{i}(v)\right]\right) \wedge z_{i}\end{aligned}\right.$ 
In particular, the first equality holds. The second identity is not needed now, but it will be used in the proof of the "general construction" theorem. In Sweedler - type notation, $\delta_{1} u=u_{1} \wedge u_{2}, \delta_{1} v=v_{1} \wedge v_{2}$, we have

$$
\begin{gathered}
0=\left[u_{1}, v\right] \wedge u_{2}+u_{1} \wedge\left[u_{2}, v\right]+\left[u, v_{1}\right] \wedge v_{2}+v_{1} \wedge\left[u, v_{2}\right] \\
=-u_{2} \wedge\left[u_{1}, v\right]+u_{1} \wedge\left[u_{2}, v\right]-v_{2} \wedge\left[u, v_{1}\right]+v_{1} \wedge\left[u, v_{2}\right] \in W \wedge \mathfrak{z}
\end{gathered}
$$

by antisymmetry of $\delta_{1}$, we have $v_{1} \wedge v_{2}=-v_{2} \wedge v_{1}$, so

$$
0=2 u_{1} \wedge\left[u_{2}, v\right]+2 v_{1} \wedge\left[u, v_{2}\right] \in W \wedge \mathfrak{z}
$$

Hence $\forall \phi \in \mathfrak{z}^{*}$ we have the cocycle formula

$$
u_{1} \phi\left(\left[v, u_{2}\right]\right)=v_{1} \phi\left(\left[u, v_{2}\right]\right)
$$

Let us introduce the following notation; for any $v \in W$ and $\phi \in \mathfrak{z}^{*}$, denote $v_{\phi} \in W^{*}$ by

$$
v_{\phi}(w):=\phi[v, w]
$$

In this notation, cocycle formula reads

$$
u_{1} v_{\phi}\left(u_{2}\right)=v_{1} u_{\phi}\left(v_{2}\right)
$$

For any $w$, apply $w_{\phi} \in W^{*}$ and get

$$
w_{\phi}\left(u_{1}\right) v_{\phi}\left(u_{2}\right)=w_{\phi}\left(v_{1}\right) u_{\phi}\left(v_{2}\right)
$$

Recall again $[,]^{*}:=\delta^{*}: W^{*} \times W^{*} \rightarrow W^{*}$ is the transpose of $\delta_{1}$, so we re-write the cocycle formula as

$$
\left[w_{\phi}, v_{\phi}\right]^{*}(u)=\left[w_{\phi}, u_{\phi}\right]^{*}(v)
$$

Now we use alternatively the antisymmetry of $[,]^{*}$ and the above formula and get

$$
\begin{aligned}
{\left[w_{\phi}, v_{\phi}\right]^{*}(u)=} & -\left[v_{\phi}, w_{\phi}\right]^{*}(u)=-\left[v_{\phi}, u_{\phi}\right]^{*}(w)=\left[u_{\phi}, v_{\phi}\right]^{*}(w) \\
& =\left[u_{\phi}, w_{\phi}\right]^{*}(v)=-\left[w_{\phi}, u_{\phi}\right]^{*}(v)
\end{aligned}
$$

hence

$$
\left[w_{\phi}, u_{\phi}\right]^{*}(v)=0 \text { for all } u, v, w \in W, \phi \in \mathfrak{z}^{*}
$$

This is almost the end of the proof. Now we simply write this formula using bases. Recall

$$
\begin{aligned}
{[\cdot, \cdot]^{*}=\sum_{k=1}^{\operatorname{dim} W} \lambda_{k} \otimes S_{k}, } & S_{i}: W^{*} \rightarrow W, & S+S^{t}=0 ; \\
{[\cdot, \cdot]=\sum_{i=1}^{\operatorname{dim} \mathfrak{z}} z_{i} \otimes T_{i}, } & T_{i}: W \rightarrow W^{*}, & T+T^{t}=0 .
\end{aligned}
$$


where $\left\{z_{i}\right\}_{i=1}^{\operatorname{dim} \mathfrak{z}}$ is basis of $\mathfrak{z}$ and $\left\{\lambda_{k}\right\}_{i=k}^{\operatorname{dim} W}$ is basis of $W^{*}$ Besides $u_{\phi}=\sum_{i=1}^{\operatorname{dim} \mathfrak{z}} \phi\left(z_{i}\right) T_{i}(u)$, so

$$
\begin{aligned}
0 & =\left[u_{\phi}, v_{\phi}\right]^{*}=\sum_{i=1}^{\operatorname{dim} \mathfrak{z}} \sum_{j=1}^{\operatorname{dim} \mathfrak{z}} \phi\left(z_{i}\right) \phi\left(z_{j}\right)\left[T_{i}(u), T_{j}(v)\right]^{*} \\
& =\sum_{i=1}^{\operatorname{dim} \mathfrak{z}} \sum_{j=1}^{\operatorname{dim} \mathfrak{z}} \sum_{k=1}^{\operatorname{dim} W} \phi\left(z_{i}\right) \phi\left(z_{j}\right) T_{j}(v)\left(S_{k}\left(T_{i}(u)\right)\right) \lambda_{k} \\
T_{j}(a)(b)=-T_{j}(b)(a) & \Rightarrow T_{j}(v)\left(S_{k}\left(T_{i}(u)\right)\right)=-T_{j}\left(S_{k}\left(T_{i}(u)\right)\right)(v), \text { so } \\
& =-\sum_{i=1}^{\operatorname{dim} \mathfrak{z}} \sum_{j=1}^{\operatorname{dim} \mathfrak{z}} \sum_{k=1}^{\operatorname{dim} W} \phi\left(z_{i}\right) \phi\left(z_{j}\right) T_{j}\left(S_{k}\left(T_{i}(u)\right)\right)(v) \lambda_{k},
\end{aligned}
$$

Since $\left\{\lambda_{k}\right\}$ is a basis

$$
0=\sum_{i, j} \phi\left(z_{i}\right) \phi\left(z_{j}\right) T_{j}\left(S_{k}\left(T_{i}(u)\right)\right)(v) \forall k
$$

Because it holds for all $u$ and $v$ we have

$$
0=\sum_{i, j} \phi\left(z_{i}\right) \phi\left(z_{j}\right) T_{j} S_{k} T_{i} \in \operatorname{Hom}\left(W, W^{*}\right)
$$

In particular, if $\left\{z^{i}\right\}$ denotes the dual basis of $\left\{z_{i}\right\}$, taking $\phi=z^{i_{0}}$ one gets

$$
0=T_{i_{0}} S_{k} T_{i_{0}} \forall k, i_{0}
$$

which is a particular case. Specializing at $\phi=z^{i_{0}}+z^{j_{0}}$ (and using the parcticular case) one can easily get

$$
0=T_{j_{0}} S_{k} T_{i_{0}}+T_{i_{0}} S_{k} T_{j_{0}} \forall i_{0}, j_{0}, k
$$

Remark 1.5. Since the Lie algebra structure will be fixed and the cobracket is the unknown, one can look at

$$
" T_{i} S_{j} T_{k}+T_{k} S_{j} T_{i}=0 \forall i, j, k "
$$

as a system of linear equations on $S$. This motivates the following definition

Definition 1.6. Keeping notation for $\mathfrak{n}=W \oplus \mathfrak{z}$ and the $T_{i}$ 's, we call $\mathfrak{n}$ a 2-step Lie algebra of TST type if the system of equations

$$
T_{i} S T_{k}+T_{k} S T_{i}=0 \forall i, k
$$

with unknown $S: W^{*} \rightarrow W$ has only the trivial solution $S=0$.

Examples of TST algebras are the Heisenberg algebras. Also one can check that $\mathfrak{f}_{n}$, the free 2-step nilpotent Lie algebra on $n$-generators, is of type TST, and this is included in a big family of examples that one can build from graphs. 
Corollary 1.7. If $(\mathfrak{n}, \delta)$ is a Lie bialgebra of type TST and $\left(\Lambda^{2} \mathfrak{n}\right)^{\mathfrak{n}}=\Lambda^{2} \mathfrak{z}$ then

- $\delta \mathfrak{z} \subset \Lambda^{2} \mathfrak{z}$

- $\delta W \subset W \wedge \mathfrak{z} \oplus \Lambda^{2} \mathfrak{z}$,

Proof. The first part is because $\delta \mathfrak{z} \subseteq\left(\Lambda^{2} \mathfrak{n}\right)^{\mathfrak{n}}$ and the second is because the $\Lambda^{2} W$-component of $\left.\delta\right|_{W}$ is $\delta_{1}$, that is determined by $S_{j}$ 's verifying the "TST" system of equations.

\subsection{General Construction}

Theorem 1.8. Assume the following data on $\mathfrak{n}=W \oplus \mathfrak{z}$ is given:

- $\delta_{\mathfrak{z}}: \mathfrak{z} \rightarrow \Lambda^{2} \mathfrak{z}$ a Lie coalgebra structure,

- a Lie algebra map $\mathbb{D}: \mathfrak{z}^{*} \rightarrow \operatorname{End}(W), f \mapsto-\sum_{i} f\left(z_{i}\right) D^{i}$ verifying the following

$$
\sum_{i} T_{i}(x)(y) \delta_{\mathfrak{z}} z_{i}=\sum_{i, j}\left(T_{i}\left(D^{j}(x)\right)(y)+T_{i}(x)\left(D^{j}(y)\right)\right) z_{i} \wedge z_{j}
$$

where $D^{i}=-\mathbb{D}\left(z^{i}\right)$.

- $\Phi: \Lambda^{2} \mathfrak{z}^{*} \rightarrow W^{*}$ a 2-cocycle with values in $W^{*}$

then the map $\delta: \mathfrak{n} \rightarrow \Lambda^{2} \mathfrak{n}$ defined by

$$
\left\{\begin{array}{lll}
\delta(z)=\delta_{\mathfrak{z}}(z) & & \text { if } z \in \mathfrak{z} \\
\delta(v)=\sum_{i} D^{i}(v) \wedge z_{i}+\Phi^{*}(v) & & \text { if } v \in W
\end{array}\right.
$$

is a Lie bialgebra structure on $\mathfrak{n}$.

Proof. Straightforward checking.

Now TST condition is a useful tool because one can easyly prove the following

Theorem 1.9. If $\mathfrak{n}$ is of type TST and $\left(\Lambda^{2} \mathfrak{n}\right)^{\mathfrak{n}}=\Lambda^{2} \mathfrak{z}$ then all Lie bialgebra structures on $\mathfrak{n}$ are as in the previous Theorem.

Proof. Last corollary says that necesarily $\delta \mathfrak{z} \subset \Lambda^{2} \mathfrak{z}$ and $\delta W \subset W \wedge \mathfrak{z} \oplus \Lambda^{2} \mathfrak{z}$. If one write $\delta$ in terms of arbitary maps $\delta_{\mathfrak{z}}, D^{j}$ s and $\Phi$, then it is a straightforward checking that co-Jacobi condition implies first and third item of Theorem 1.8 and the 1-cocycle condition implies the second item.

\section{Graph algebras}

Let $G=(V, A)$ be an oriented simple graph without loops. The graph algebra $\mathfrak{n}(G)$ associated to a graph $G$ is defined in the following way: for each $i, j \in V$ and $\alpha \in A$ going from $i$ to $j$ we set

$$
\left[e_{i}, e_{j}\right]:=\alpha
$$

Since the isomorphism class do not depends on the orientation (just change $\alpha$ by $-\alpha$ ), sometimes we will assume that $G$ is unoriented, but the set of vertices is ordered, so that an edge joining two vertices can be oriented, considering that it goes from the smaller to the bigger. 
Examples 2.1. $\quad$ 1. the graph $x \stackrel{Z}{\longrightarrow} y$ gives the 3-dimensional Lie algebra with basis $\{x, y, z\}$ and bracket $[x, y]=z$, that is, the Heisenberg algebra $\mathfrak{h}_{3}$. On the other hand, for $n>1$ the Heisenberg Lie algebra $\mathfrak{h}_{2 n+1}$ is not a graph algebra.

2. $\mathfrak{f}_{n}$ : the free 2-step nilpotent Lie algebra is the graph algebra associated to the complete graph $K_{n}$.

3. The nontrivial brackets of the Lie algebra associated to the following graph are

$$
\begin{gathered}
\underbrace{e_{2}}_{e_{1} \underset{\beta}{\longrightarrow} e_{3} e^{2}} e_{4} \\
{\left[e_{1}, e_{2}\right]=\gamma, \quad\left[e_{1}, e_{3}\right]=\beta,} \\
{\left[e_{2}, e_{3}\right]=\alpha, \quad\left[e_{4}, e_{3}\right]=\rho .}
\end{gathered}
$$

Notice that the center of a graph algebra has natural basis consisting on the arrows and the isolated vertices. If we assume that the graph does not have isolated vertices then we have canonical decomposition and basis:

$$
W=\bigoplus_{e \in V} k e, \mathfrak{z}=\bigoplus_{\alpha \in A} k \alpha
$$

and $\mathfrak{n}(G)=W \oplus \mathfrak{z}$ is a 2-step nilpotent with center $\mathfrak{z}$ and linear complement $W$.

We begin by characterizing the condition $\left(\Lambda^{2} \mathfrak{n}\right)^{\mathfrak{n}}=\Lambda^{2} \mathfrak{z}$. Recall that for a vertex $e$, the degree - or valency- $|e|$ is the number of edges incident to $e$.

Lemma 2.2. Consider a graph $G=(V, A)$ such that there exists $e \in V$ with $|e|=1$ then there exists an element $0 \neq \omega \in(W \wedge \mathfrak{z})^{\mathfrak{n}}$.

Proof. Let $\alpha$ be the unique edge joining $e$ with $e^{\prime}$,

If $\omega:=e \wedge \alpha \in W \wedge \mathfrak{z}$ then $\operatorname{ad}_{e^{\prime}} \omega= \pm \alpha \wedge \alpha=0$, and clearly also $\operatorname{ad}_{e^{\prime \prime}} \omega=0$ for any other $e^{\prime \prime} \in V$.

A more involve prove is needed for the following Lemma:

Lemma 2.3. Let $\omega \in(W \wedge \mathfrak{z})^{\mathfrak{n}}$, if $\omega=\sum_{e \in V, \alpha_{i} \in A} \lambda_{e, \alpha} e \wedge \alpha_{i}$, then $\lambda_{e, \alpha}=0$ for all $\alpha \in A$ and for each $e \in V$ such that $|e| \geq 2$.

Proof. Consider $e \in V$ a vertex with $|e| \geq 2$.

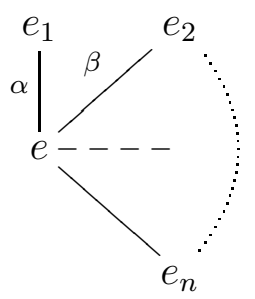


Denote $e^{\prime}=e_{1}$ and $e^{\prime \prime}=e_{2}$ in the drawing, i.e. $e^{\prime}$ and $e^{\prime \prime}$ are two different vertices incident to $e$ with corresponding edges $\alpha$ and $\beta$. A general element in $W \wedge \mathfrak{z}$ will be of the form

$$
\begin{aligned}
& \omega=\quad \text { ae } \wedge \alpha \quad+b e \wedge \beta \quad+e \wedge\left(\sum_{\alpha_{i} \neq \alpha, \beta} \lambda_{i} \alpha_{i}\right) \\
& +a^{\prime} e^{\prime} \wedge \alpha \quad+b^{\prime} e^{\prime} \wedge \beta \quad+e^{\prime} \wedge\left(\sum_{\alpha_{i} \neq \alpha, \beta}^{\alpha_{i} \neq \alpha, \beta} \mu_{i} \alpha_{i}\right) \\
& +a^{\prime \prime} e^{\prime \prime} \wedge \alpha \quad+b^{\prime \prime} e^{\prime \prime} \wedge \beta \quad+e^{\prime \prime} \wedge\left(\sum_{\alpha_{i} \neq \alpha, \beta} \nu_{i} \alpha_{i}\right) \\
& +\sum_{e^{\prime \prime \prime} \neq e, e^{\prime}, e^{\prime \prime}} a_{e^{\prime \prime \prime}} e^{\prime \prime \prime} \wedge \alpha+\sum_{e^{\prime \prime \prime} \neq e, e^{\prime}, e^{\prime \prime}} b_{e^{\prime \prime \prime}} e^{\prime \prime \prime} \wedge \beta+\sum_{\substack{e^{\prime \prime \prime} \neq e, e^{\prime}, e^{\prime \prime} \\
\alpha_{i} \neq \alpha, \beta}} \lambda_{i, e^{\prime \prime \prime}} e^{\prime \prime \prime} \wedge \alpha_{i}
\end{aligned}
$$

for some $a, a^{\prime}, a^{\prime \prime} a_{i}, \mu_{i}, \nu_{i}, \lambda_{i, e^{\prime \prime \prime}} \in k$. Since $\omega$ is supposed to be invariant, we have

$$
\begin{aligned}
& 0=\operatorname{ad}_{e} \omega=0 \\
& +a^{\prime} \alpha \wedge \alpha+b^{\prime} \alpha \wedge \beta \\
& +a^{\prime \prime} \beta \wedge \alpha \quad+b^{\prime \prime} \beta \wedge \beta \\
& +\sum_{e^{\prime \prime \prime} \neq e, e^{\prime}, e^{\prime \prime}} a_{e^{\prime \prime \prime}}\left[e, e^{\prime \prime \prime}\right] \wedge \alpha+\sum_{e^{\prime \prime \prime} \neq e, e^{\prime}, e^{\prime \prime}} b_{e^{\prime \prime \prime}}\left[e, e^{\prime \prime \prime}\right] \wedge \beta+\sum_{\substack{e^{\prime \prime \prime} \neq e, e^{\prime}, e^{\prime \prime} \\
\alpha_{i} \neq \alpha, \beta}} \lambda_{i, e^{\prime \prime \prime}}\left[e, e^{\prime \prime \prime}\right] \wedge \alpha_{i}
\end{aligned}
$$

then, in particular $b^{\prime}=a^{\prime \prime} \quad(*)$.

Analogously, compute $0=\operatorname{ad}_{e^{\prime}} \omega=$

$$
\begin{array}{ccc}
-a \alpha \wedge \alpha & -b \alpha \wedge \beta & -\alpha \wedge\left(\sum_{\substack{\alpha_{i} \neq \alpha, \beta \\
+0}} \lambda_{i} \alpha_{i}\right) \\
+a^{\prime \prime}\left[e^{\prime}, e^{\prime \prime}\right] \wedge \alpha & +0 & +0 \\
+\sum_{e^{\prime \prime \prime} \neq e, e^{\prime}, e^{\prime \prime}} a_{e^{\prime \prime \prime}}\left[e^{\prime}, e^{\prime \prime \prime}\right] \wedge \alpha & +\sum_{e^{\prime \prime \prime} \neq e, e^{\prime}, e^{\prime \prime}} b_{e^{\prime \prime \prime}}\left[e^{\prime}, e^{\prime \prime \prime}\right] \wedge \beta & +\sum_{\substack{e^{\prime \prime \prime} \neq e, e^{\prime}, e^{\prime \prime} \\
\alpha_{i} \neq \alpha, \beta}} \lambda_{i, e^{\prime \prime \prime}}\left[e^{\prime}, e^{\prime \prime \prime}\right] \wedge \alpha_{i} \\
= & -b \alpha \wedge \beta & -\alpha \wedge\left(\sum_{\alpha_{i} \neq \alpha, \beta} \lambda_{i} \alpha_{i}\right) \\
+\sum_{e^{\prime \prime \prime} \neq e, e^{\prime}, e^{\prime \prime}} a_{e^{\prime \prime \prime}}\left[e^{\prime}, e^{\prime \prime \prime}\right] \wedge \alpha & +\sum_{e^{\prime \prime \prime} \neq e, e^{\prime}, e^{\prime \prime}} b_{e^{\prime \prime \prime}}\left[e^{\prime}, e^{\prime \prime \prime}\right] \wedge \beta & +\sum_{\substack{\sum^{\prime \prime \prime} \neq e, e^{\prime}, e^{\prime \prime} \\
\alpha_{i} \neq \alpha, \beta}} \lambda_{i, e^{\prime \prime \prime}}\left[e^{\prime}, e^{\prime \prime \prime}\right] \wedge \alpha_{i}
\end{array}
$$

Hence, $b=0$. In the same way $0=\operatorname{ad}_{e^{\prime \prime}} \omega=$

$$
\begin{array}{ccc}
-a \beta \wedge \alpha & -\beta \wedge\left(\sum_{\alpha_{i} \neq \alpha, \beta} \lambda_{i} \alpha_{i}\right) \\
+a^{\prime}\left[e^{\prime \prime}, e^{\prime}\right] \wedge \alpha & +b^{\prime}\left[e^{\prime \prime}, e^{\prime}\right] \wedge \beta & +\left[e^{\prime \prime}, e^{\prime}\right] \wedge\left(\sum_{\alpha_{i} \neq \alpha, \beta} \nu_{i} \alpha_{i}\right) \\
+\sum_{e^{\prime \prime \prime} \neq e, e^{\prime}, e^{\prime \prime}} a_{e^{\prime \prime \prime}}\left[e^{\prime \prime}, e^{\prime \prime \prime}\right] \wedge \alpha & +\sum_{e^{\prime \prime \prime} \neq e, e^{\prime}, e^{\prime \prime}} b_{e^{\prime \prime \prime}}\left[e^{\prime \prime}, e^{\prime \prime \prime}\right] \wedge \beta & +\sum_{\substack{e^{\prime \prime \prime} \neq e, e^{\prime}, e^{\prime \prime} \\
\alpha_{i} \neq \alpha, \beta}} \lambda_{i, e^{\prime \prime \prime}}\left[e^{\prime \prime}, e^{\prime \prime \prime}\right] \wedge \alpha_{i}
\end{array}
$$

Hence, $a=0$. To continue, we need to consider the following cases $i$ ) and $i i$ ). 
i) Suppose $e^{\prime}$ and $e^{\prime \prime}$ are not joined by any edge in the graph, so $\left[e^{\prime}, e^{\prime \prime}\right]=0$, then (1) equals

$$
\begin{gathered}
0=\left(\sum_{\alpha_{i} \neq \alpha, \beta} \lambda_{i} \alpha_{i}+\sum_{e^{\prime \prime \prime} \neq e, e^{\prime}, e^{\prime \prime}} a_{e^{\prime \prime \prime}}\left[e^{\prime}, e^{\prime \prime \prime}\right]\right) \wedge \alpha \\
+\sum_{e^{\prime \prime \prime} \neq e, e^{\prime}, e^{\prime \prime}} b_{e^{\prime \prime \prime}}\left[e^{\prime}, e^{\prime \prime \prime}\right] \wedge \beta \quad+\sum_{\substack{e^{\prime \prime \prime} \neq e, e^{\prime}, e^{\prime \prime} \\
\alpha_{i} \neq \alpha, \beta}} \lambda_{i, e^{\prime \prime \prime}}\left[e^{\prime}, e^{\prime \prime \prime}\right] \wedge \alpha_{i}
\end{gathered}
$$

Notice in the previous equation that all the terms belong to different components, so $\left(1^{\prime}\right)$ implies $\lambda_{i}=0$ for all $i$ except those corresponding to $\alpha_{i}$ incident to $e^{\prime}$.

In the same way, (2) implies $\lambda_{i}=0$ for all $i$ except those corresponding to $\alpha_{i}$ incident to $e^{\prime \prime}$. But there is no edge between $e^{\prime}$ and $e^{\prime \prime}$, hence all $\lambda_{i}=0$.

ii) Suppose $e^{\prime}$ and $e^{\prime \prime}$ are joined by an edge $\gamma$, so $\left[e^{\prime}, e^{\prime \prime}\right]=\gamma$. Equation 1 reads in this case

$$
\begin{aligned}
0=\left(\sum_{\alpha_{i} \neq \alpha, \beta} \lambda_{i} \alpha_{i}+a^{\prime \prime} \gamma+\sum_{e^{\prime \prime \prime} \neq e, e^{\prime}, e^{\prime \prime}} a_{e^{\prime \prime \prime}}\left[e^{\prime}, e^{\prime \prime \prime}\right]\right) \wedge \alpha & \\
+b^{\prime \prime} \gamma \wedge \beta & +\gamma \wedge\left(\sum_{\alpha_{i} \neq \alpha, \beta} \nu_{i} \alpha_{i}\right) \\
+\sum_{e^{\prime \prime \prime} \neq e, e^{\prime}, e^{\prime \prime}} b_{e^{\prime \prime \prime}}\left[e^{\prime}, e^{\prime \prime \prime}\right] \wedge \beta & +\sum_{\substack{\alpha^{\prime \prime \prime} \neq e, e^{\prime}, e^{\prime \prime \prime} \\
\alpha_{i} \neq \alpha, \beta}} \lambda_{i, e^{\prime \prime \prime}}\left[e^{\prime}, e^{\prime \prime \prime}\right] \wedge \alpha_{i}
\end{aligned}
$$

Looking at the terms with common factor $\alpha$, we see that $\lambda_{i}=0$ for all $i$ except those corresponding to $\alpha_{i}$ incident to $e^{\prime}$. A similar computation interchanging $e^{\prime}$ with $e^{\prime \prime}$ says $\lambda_{i}=0$ for all $i$ except those corresponding to $\alpha_{i}$ incident to $e^{\prime \prime}$.

Resuming, if we call $c=b^{\prime}=a^{\prime \prime}$ and $\lambda=\lambda_{\gamma}, \omega$ is of the following form

$$
\begin{array}{rlrl}
\omega= & +c e^{\prime} \wedge \beta & & e \wedge \lambda \gamma \\
+a^{\prime} e^{\prime} \wedge \alpha & +e^{\prime} \wedge\left(\sum_{\substack{\alpha_{i} \neq \alpha, \beta \\
\mu_{i}}} \mu_{i}\right) \\
+c e^{\prime \prime} \wedge \alpha & +b^{\prime \prime} e^{\prime \prime} \wedge \beta & +e^{\prime \prime} \wedge\left(\sum_{\alpha_{i} \neq \alpha, \beta} \nu_{i} \alpha_{i}\right) \\
+\sum_{e^{\prime \prime \prime} \neq e, e^{\prime}, e^{\prime \prime}} a_{e^{\prime \prime \prime}} e^{\prime \prime \prime} \wedge \alpha & +\sum_{e^{\prime \prime \prime} \neq e, e^{\prime}, e^{\prime \prime}} b_{e^{\prime \prime \prime}} e^{\prime \prime \prime} \wedge \beta & +\sum_{\substack{e_{i}^{\prime \prime \prime} \neq e, e^{\prime}, e^{\prime \prime} \\
\alpha_{i} \neq \alpha, \beta}} \lambda_{i, e^{\prime \prime \prime}} e^{\prime \prime \prime} \wedge \alpha_{i}
\end{array}
$$

We go back to equations (1) and (2), we have

$$
\begin{array}{ccc}
0= & -\lambda \alpha \wedge \gamma \\
+c \gamma \wedge \alpha & +b^{\prime \prime} \gamma \wedge \beta & +\gamma \wedge\left(\sum_{\alpha_{i} \neq \alpha, \beta} \nu_{i} \alpha_{i}\right) \\
+\sum_{e^{\prime \prime \prime} \neq e, e^{\prime}, e^{\prime \prime}} a_{e^{\prime \prime \prime}}\left[e^{\prime}, e^{\prime \prime \prime}\right] \wedge \alpha & +\sum_{e^{\prime \prime \prime} \neq e, e^{\prime}, e^{\prime \prime}} b_{e^{\prime \prime \prime}}\left[e^{\prime}, e^{\prime \prime \prime}\right] \wedge \beta & +\sum_{\substack{e^{\prime \prime \prime} \neq e, e^{\prime}, e^{\prime \prime} \\
\alpha_{i} \neq \alpha, \beta}} \lambda_{i, e^{\prime \prime \prime}}\left[e^{\prime}, e^{\prime \prime \prime}\right] \wedge \alpha_{i}
\end{array}
$$


and

$0=$ $-\lambda \beta \wedge \gamma$

$$
\begin{array}{ccc}
-a^{\prime} \gamma \wedge \alpha & -c \gamma \wedge \beta & -\gamma \wedge\left(\sum_{\alpha_{i} \neq \alpha, \beta} \nu_{i} \alpha_{i}\right) \\
+\sum_{e^{\prime \prime \prime} \neq e, e^{\prime}, e^{\prime \prime}} a_{e^{\prime \prime \prime}}\left[e^{\prime \prime}, e^{\prime \prime \prime}\right] \wedge \alpha & +\sum_{e^{\prime \prime \prime} \neq e, e^{\prime}, e^{\prime \prime}} b_{e^{\prime \prime \prime}}\left[e^{\prime \prime}, e^{\prime \prime \prime}\right] \wedge \beta & +\sum_{\substack{\prime \prime \prime \neq e, e^{\prime}, e^{\prime \prime} \\
\alpha_{i} \neq \alpha, \beta}} \lambda_{i, e^{\prime \prime \prime}}\left[e^{\prime \prime}, e^{\prime \prime \prime}\right] \wedge \alpha_{i}
\end{array}
$$

We look at the terms with $\alpha \wedge \gamma$ in (1) and $\beta \wedge \gamma$ in (2) and obtain

$$
\lambda+c=0 \text { and } \lambda-c=0
$$

Hence, $\lambda=0$, so $\lambda_{e, \alpha_{i}}=0$ for all $\alpha_{i} \in A$.

As a corollary we can prove the following characterization:

Theorem 2.4. For a graph algebra (without isolated vertices), $\left(\Lambda^{2} \mathfrak{n}\right)^{\mathfrak{n}}=\Lambda^{2} \mathfrak{z}$ if and only if $|e| \geq 2$ for all $e \in V$.

\subsection{Graph algebras and the TST equations}

For a graph $(G=(V, A)$ with vertices $V$ and arrows $A$ we use the canonical basis of the center $\left\{z_{i}\right\}_{i=1}^{\operatorname{dim} \mathfrak{z}}=\{\alpha\}_{\alpha \in \in A}$. The system of equations with unknown antisymmetric $\operatorname{map} S: W^{*} \rightarrow W$ is of the form

$$
T_{\alpha} S T_{\beta}+T_{\beta} S T_{\alpha}=0 \forall \alpha, \beta \in A
$$

Fix $V=\left\{e_{1}, \ldots, e_{n}\right\}$ the set of vertices, it is a basis of $W$ by definition; let $\left\{e_{1}^{*}, \ldots, e_{n}^{*}\right\}$ be the dual basis. It is easy to see that for each $\alpha \in A$ joining $e_{i}$ with $e_{j}$, with $i<j$ we have

$$
T_{\alpha}\left(e_{i}\right)=e_{j}^{*}, T_{\alpha}\left(e_{j}\right)=-e_{i}^{*}, T_{\alpha}\left(e_{k}\right)=0 \forall k \neq i, j
$$

In matrix notation $\left[T_{\alpha}\right]=E_{j, i}-E_{i, j}$. The following is a translation of the TST condition in graph language:

Theorem 2.5. Let $\mathfrak{n}$ be a 2-steps nilpotent Lie algebra arising from a graph $G=(V, A)$, $i, j \in V$ and $S$ an antisymmetric solution of the system $T_{\alpha} S T_{\beta}+T_{\beta} S T_{\alpha}=0 \forall \alpha, \beta \in A$.

1. If there exists an edge $\alpha$ joining $i$ and $j$, then $S_{i, j}=0$.

$$
i \stackrel{\alpha}{\longrightarrow} j
$$

2. If there are two edges $\alpha$ and $\beta$ and four vertices $i, i^{\prime}, j, j^{\prime}$ such that $\alpha$ joins $i$ and $i^{\prime}$ and $\beta$ joins $j$ and $j^{\prime}$ with $\left\{i, i^{\prime}\right\} \cap\left\{j, j^{\prime}\right\}=\emptyset$ then $S_{i, j}=0$.

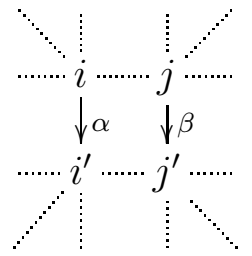


Proof. Consider the equation $T_{\alpha} S T_{\alpha}=0$ for a given $\alpha \in A$, where $\alpha$ joins $e_{i}$ with $e_{j}$.

$$
\left(T_{\alpha} S T_{\alpha}\right)_{i, j}=\sum_{k, \ell}\left(T_{\alpha}\right)_{i, k}(S)_{k, \ell}\left(T_{\alpha}\right)_{\ell, j}=\left(T_{\alpha}\right)_{i, j}(S)_{j, i}\left(T_{\alpha}\right)_{i, j}=(S)_{j, i}
$$

This shows 1 . Next suppose we have two edges $\alpha$ and $\beta$ such that $\alpha$ joins $i$ and $i^{\prime}$, and $\beta$ joins $j$ and $j^{\prime}$ with $\left\{i, i^{\prime}\right\} \cap\left\{j, j^{\prime}\right\}=\emptyset$ then $S_{i, j}=0$. Writing the TST- equation for these $\alpha, \beta$,

$$
\begin{aligned}
\left(T_{\alpha} S T_{\beta}+T_{\beta} S T_{\alpha}\right)_{i^{\prime}, j^{\prime}} & =\sum_{k, \ell}\left(T_{\alpha}\right)_{i^{\prime}, k}(S)_{k, \ell}\left(T_{\beta}\right)_{\ell, j^{\prime}}+\sum_{k, \ell}\left(T_{\beta}\right)_{i^{\prime}, k}(S)_{k, \ell}\left(T_{\alpha}\right)_{\ell, j^{\prime}} \\
& =\left(T_{\alpha}\right)_{i^{\prime}, i}(S)_{i, j}\left(T_{\beta}\right)_{j, j^{\prime}}= \pm(S)_{i, j}
\end{aligned}
$$

since in the second sum, $\left(T_{\beta}\right)_{i^{\prime}, k}=0=\left(T_{\alpha}\right)_{\ell, j^{\prime}}$ for all $k, \ell$.

\section{Examples}
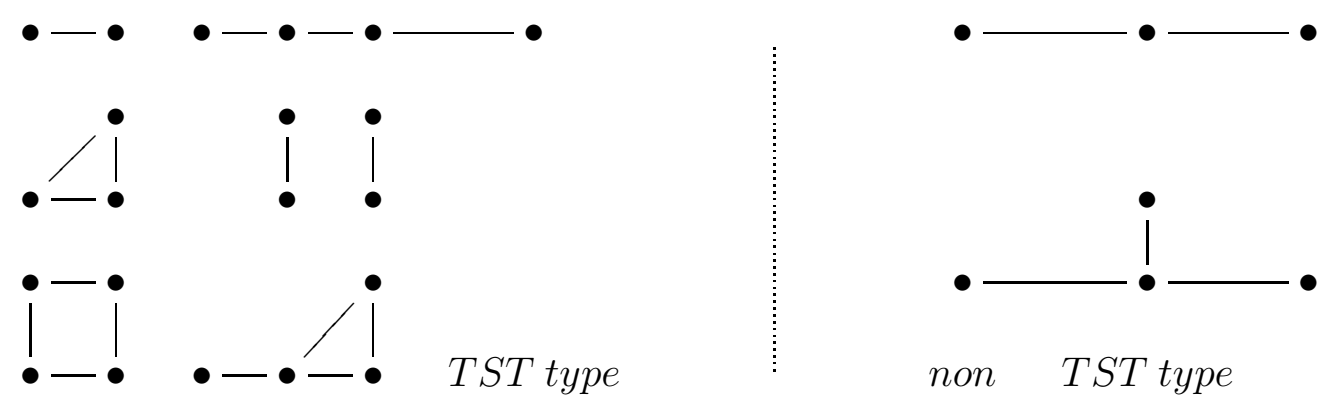

non TST type

Remark 2.6. If we are interested in algebras with $\left(\Lambda^{2} \mathfrak{n}\right)^{\mathfrak{n}}=\Lambda^{2} \mathfrak{z}$ then we must look at graphs with $|e| \geq 2$ for all $e \in V$. But if this is the case, then any pair $i, j$ in $V$ satisfies either condition 1 or 2 of the previous proposition, so we have the following corollary:

Corollary 2.7. If $|e| \geq 2$ for all $e \in V$ then $\left(\Lambda^{2} \mathfrak{n}\right)^{\mathfrak{n}}=\Lambda^{2} \mathfrak{z}$ and also $\mathfrak{n}$ is of TST type, as a consequence, any cobracket structure on $\mathfrak{n}$ satisfies

$$
\delta \mathfrak{z} \subseteq \Lambda^{2} \mathfrak{z}, \quad \delta W \subseteq W \wedge \mathfrak{z} \oplus \Lambda^{2} \mathfrak{z},
$$

and it is of the form as in Theorem 1.8

Remark 2.8. In the case where a graph has (all vertices with) valency bigger or equal to 2, even if the above corollary shows a big simplification of the structure constants, in order to find all possible data as in Theorem 1.8 we still have to solve nonlinear equations, for instance, the data includes a Lie algebra structure on $\mathfrak{z}^{*}$. We present next a family of Lie bialgebras that include the ones where the 2-cocycle $\delta$ is a coboundary where this part of the data is trivial. 


\section{Nearly Coboundary Lie bialgebras}

Recall that a Lie bialgebra $(\mathfrak{n}, \delta)$ is called coboundary if there exists $r \in \Lambda^{2} \mathfrak{n}$ such that

$$
\delta(x)=\operatorname{ad}_{x}(r) \forall x \in \mathfrak{n}
$$

In any coboudary bialgebra we have $\delta(\mathfrak{z})=0$. This motivates the following definition

Definition 3.1. A Lie bialgebra $(\mathfrak{n}, \delta)$ will be called nearly coboundary if $\left.\delta\right|_{\mathfrak{z}} \equiv 0$.

Example 3.2. Let $\delta$ satisfying $\delta(W) \subset \Lambda^{2} \mathfrak{z}$, that is, it is of the form

$$
\delta\left(e_{i}\right)=\sum_{\alpha, \beta \in A} \mu_{i}^{\alpha, \beta} \alpha \wedge \beta \quad \forall i \in V
$$

with arbitrary coefficients $\mu_{i}^{\alpha, \beta} \in K$ verifing only $\mu_{i}^{\alpha, \beta}=-\mu_{i}^{\beta, \alpha}$ for all $i, \alpha, \beta$. Then $\delta$ endows $\mathfrak{n}$ of a nearly coboundary Lie bialgebra structure. Notice that there are $|V| \frac{|A|(|A|-1)}{2}$ free parameters, while for a coboundary Lie bialgebra structure with $\delta(W) \subset$ $\Lambda^{2} \mathfrak{z}$ we need an element

$$
r=\sum_{i \in V, \alpha \in A} r_{i j} e_{i} \wedge \alpha \in W \wedge \mathfrak{z}
$$

In order to give $r$ we need $|V||A|$ parameters, and for $|A|>3$ it is clear that $|V| \frac{|A|(|A|-1)}{2}>$ $|V||A|$, so in particular there is a lot non coboundary Lie algebras in this family of examples.

As particular cases, for $G=C_{n}$ we have $|V|=n$ and $|A|=n$ and and $G=K_{n}$, $\mid V=n$ and $\mid A=n(n-1) / 2$. Except $n=3$, there are a lot of nearly coboundary Lie

\begin{tabular}{|c|c|c|c|c|}
\hline & $\begin{array}{c}C_{n} \\
\mid V \| A\end{array}$ & $|V| \frac{|A|(|A|-1)}{2}$ & $\begin{array}{c}K_{n} \\
|V||A|\end{array}$ & $|V| \frac{|A|(|A|-1)}{2}$ \\
\hline$n$ & $n^{2}$ & $\frac{n^{2}(n-1)}{2}$ & $\frac{n^{2}(n-1)}{2}$ & $\frac{1}{8} n^{2}(n+1)(n-1)(n-2)$ \\
\hline 3 & 9 & 9 & 9 & 9 \\
\hline 4 & 16 & 24 & 24 & 60 \\
\hline 5 & 25 & 50 & 50 & 225 \\
\hline 6 & 36 & 90 & 90 & 630 \\
\hline
\end{tabular}
bialgebras of this type that are not coboundary. In the table we write the numbers $|V| \frac{|A|(|A|-1)}{2}$ and $|V||A|$ in general and for small $n$ :

Now we will study conditions of Theorem [?] for nearly coboundary bialgebra structures on graph algebras $\mathfrak{n}=\mathfrak{n}(G)$.

Lemma 3.3. Let $G$ be a graph with $|e| \geq 2 \forall e \in V, \mathfrak{n}=\mathfrak{n}(G), \delta: \mathfrak{n} \rightarrow \Lambda^{2} \mathfrak{n}$ a Lie bialgebra structure. Assume that $\delta$ is nearly coboundary, so that $\delta(z)=0$ if $z \in \mathfrak{z}$. If we write (as in Theorem 1.8)

$$
\delta(v)=\sum_{\alpha \in A} D_{\alpha}(v) \wedge \alpha+\Phi^{*}(v) \text { if } v \in W
$$

then $D_{\alpha} D_{\beta}=D_{\beta} D_{\alpha}$ for all edges $\alpha, \beta$. 
Proof. We know that all bialgebra structures are as in Theorem 1.8, so they must satisfy

$$
\sum_{\alpha, \in A} D_{\alpha}\left(e_{i}\right) \wedge \delta(\alpha)=\sum_{\alpha, \beta \in A} D_{\alpha}\left(D_{\beta}\left(e_{i}\right)\right) \wedge \alpha \wedge \beta
$$

But because $\delta(\mathfrak{z})=0$ we have

$$
0=\sum_{\alpha, \beta \in A} D_{\alpha}\left(D_{\beta}\left(e_{i}\right)\right) \wedge \alpha \wedge \beta=\sum_{\alpha<\beta \in A}\left(D_{\alpha} D_{\beta}-D_{\beta} D_{\alpha}\right)\left(e_{i}\right) \wedge \alpha \wedge \beta
$$

So $D_{\alpha} D_{\beta}=D_{\beta} D_{\alpha}$.

From the above lemma, we see that a typical situation will be when the $D_{\alpha}{ }^{\prime}$ s are simultaneously diagonalizable. In next subsection we study this particular case.

\subsection{Nearly coboundary bialgebras with diagonalizable $D_{\alpha}$ 's}

In this subsection we suppose that all the $\left\{D_{\alpha}: \alpha \in A\right\}$ are simultaneously diagonalizable, but moreover that the set of vertices $\left\{e_{i}: 1 \leq i \leq n\right\}=V$ is a basis of eigenvectors. We denote by $\lambda_{i, \alpha}$ the corresponding eigenvalues, i.e., $\forall i, \alpha$

$$
D_{\alpha}\left(e_{i}\right)=\lambda_{i, \alpha} e_{i}
$$

Remark 3.4. The assumption that the set of vertices are eigenvalues is not a lost of generality in the family of free 2-step nilpotent algebras.

Proposition 3.5. Let $\alpha_{0}$ be an edge joining $i_{0}$ with $j_{0}$, then

$$
\lambda_{i_{0}, \alpha}=-\lambda_{j_{0}, \alpha} \forall \alpha \neq \alpha_{0}
$$

Proof. From the cocycle condition, the assumption $\delta(\mathfrak{z})=0$ and $\delta_{W}\left(e_{i_{0}}\right)=\sum_{\alpha \in A} D_{\alpha}\left(e_{i_{0}}\right) \wedge$ $\alpha$, we have

$$
\begin{gathered}
0=\delta\left(\alpha_{0}\right)=\delta\left(\left[e_{i_{0}}, e_{j_{0}}\right]\right)=\left[\delta\left(e_{i_{0}}\right], e_{j_{0}}\right]+\left[e_{i_{0}}, \delta\left(e_{j_{0}}\right)\right] \\
=\left[\sum_{\alpha \in A} D_{\alpha}\left(e_{i_{0}}\right) \wedge \alpha, e_{j_{0}}\right]+\left[e_{i_{0}}, \sum_{\alpha \in A} D_{\alpha}\left(e_{j_{0}}\right) \wedge \alpha\right] \\
=\left[\sum_{\alpha \in A} \lambda_{i_{0}, \alpha} e_{i_{0}} \wedge \alpha, e_{j_{0}}\right]+\left[e_{i_{0}}, \sum_{\alpha \in A} \lambda_{j_{0}, \alpha} e_{j_{0}} \wedge \alpha\right] \\
=\alpha_{0} \wedge\left(\sum_{\alpha \in A} \lambda_{i_{0}, \alpha}+\lambda_{j_{0}, \alpha}\right) \alpha
\end{gathered}
$$

then each coefficient $\lambda_{i_{0}, \alpha}+\lambda_{j_{0}, \alpha}=0$ for all $\alpha \neq \alpha_{0}$.

Example 3.6. For the graph

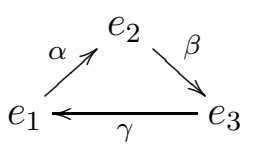


we get $f_{3}$, the free 2 -step nilpotent Lie algebra on 3 generators $\left\{e_{1}, e_{2}, e_{3}\right\}$. The complete list of Lie bialgebra structures on $\mathfrak{f}_{3}$ such that $\delta(\mathfrak{z})=0$ and diagonal $D_{\alpha_{i}}$ for all $i=1,2,3$ is

$$
\begin{gathered}
\delta\left(e_{1}\right)=e_{1} \wedge(a \alpha+b \beta+c \gamma)+\omega_{1}=: e_{1} \wedge A_{1}+\omega_{1} \\
\delta\left(e_{2}\right)=e_{2} \wedge(a \alpha-b \beta-c \gamma)+\omega_{2}=: e_{2} \wedge A_{2}+\omega_{2} \\
\delta\left(e_{3}\right)=e_{3} \wedge(-a \alpha-b \beta+c \gamma)+\omega_{3}=: e_{3} \wedge A_{3}+\omega_{3}
\end{gathered}
$$

for any $a, b, c \in k, \omega_{i} \in \Lambda^{2} \mathfrak{z}$ satisfying co - Jacobi condition:

$$
\omega_{i} \wedge A_{i}=0, \quad i=1,2,3
$$

For highly connected graphs the situation is even more favorable:

Proposition 3.7. Consider a graph $G=(V, A)$, a vertex $i_{0} \in V$ and $\alpha \in A$. If there exists a path in $G$ of the forms

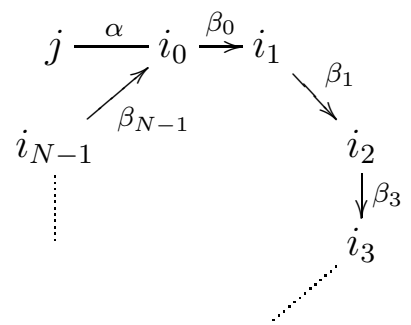

or

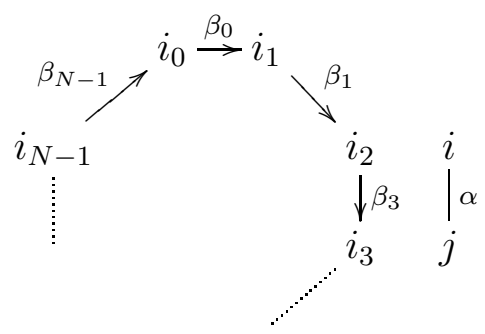

then $\lambda_{i_{0}, \alpha}=(-1)^{N} \lambda_{i_{0}, \alpha}$; if $N$ is odd then $\lambda_{i_{0}, \alpha}=0$

As a corollary we have

Corollary 3.8. If $n \geq 4$ and $\delta$ is a bialgebra structure on $\mathfrak{f}_{n}$ satisfying $\delta(\mathfrak{z})=0$ and $D_{\alpha}$ diagonalizable for all $\alpha$, then

$$
\delta(W) \subset \Lambda^{2} \mathfrak{z},
$$

that is, all $D_{\alpha}$ are necessarily zero and $\delta\left(e_{i}\right)$ is of the form $\delta\left(e_{i}\right)=\omega_{i}$ with arbitrary $\omega_{i} \in \Lambda^{2} \mathfrak{z}$.

Proof. if $i_{0}$ is a vertex and $\alpha$ an edge, in the complete graph on $n$ vertices with $n \geq 4$ we are always in one of the following situations:

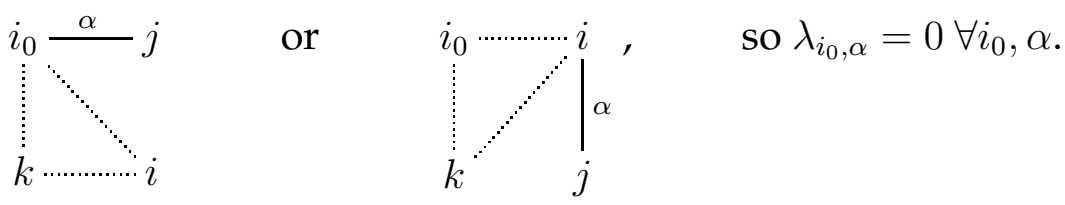

We finish exhibiting examples of non diagonalizable $D_{i}{ }^{\prime}$ s in $\mathfrak{f}_{3}$. We do not know if there are similar nontrivial examples in $\mathfrak{f}_{n}$ for $n \geq 4$. 


\subsection{Non-diagonalizable $D^{\prime}$ s in $\mathfrak{f}_{3}$}

We fix the notation

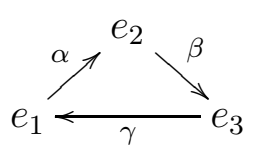

Since $\left[D_{\alpha_{i}}, D_{\alpha_{j}}\right]=0$ we may always assume that there exists a basis where all $D_{i}$ are simultaneously upper triangular, that is, of the form $\left(\begin{array}{lll}a & b & c \\ 0 & d & e \\ 0 & 0 & f\end{array}\right)$.

But also, if one of them has three different elements in the diagonal, then all of them are diagonalizable, and we are in the previous case. So we may assume that all of them have some multiplicity on the eigenvalues. We slit in two cases: multiplicity 2 and multiplicity 3 . In multiplicity 3 there are two possibilities: there is a $D$ with a single Jordan block of size 3 , or the maximal size of the Jordan block is 2 . Writing the first $D$ in Jordan form and using that the other $D^{\prime}$ s commute with this one we arrive at the following cases:

Multiplicity 2:

$$
D_{\alpha}=\left(\begin{array}{ccc}
\lambda & 1 & 0 \\
0 & \lambda & 0 \\
0 & 0 & \lambda^{\prime}
\end{array}\right), D_{\beta}=\left(\begin{array}{ccc}
a & b & 0 \\
0 & a & 0 \\
0 & 0 & c
\end{array}\right), D_{\gamma}=\left(\begin{array}{ccc}
\mu & \nu & 0 \\
0 & \mu & 0 \\
0 & 0 & \tau
\end{array}\right)
$$

where $\lambda \neq \lambda^{\prime}$, and multiplicity 3:

$$
D_{\alpha}=\left(\begin{array}{ccc}
\lambda & 1 & 0 \\
0 & \lambda & 1 \\
0 & 0 & \lambda
\end{array}\right), D_{\beta}=\left(\begin{array}{ccc}
a & b & c \\
0 & a & b \\
0 & 0 & a
\end{array}\right), D_{\gamma}=\left(\begin{array}{ccc}
\mu & \nu & \rho \\
0 & \mu & \nu \\
0 & 0 & \mu
\end{array}\right)
$$

or

$$
D_{\alpha}=\left(\begin{array}{ccc}
\lambda & 1 & 0 \\
0 & \lambda & 0 \\
0 & 0 & \lambda
\end{array}\right), D_{\beta}=\left(\begin{array}{ccc}
a & b & c \\
0 & a & 0 \\
0 & 0 & a
\end{array}\right), D_{\gamma}=\left(\begin{array}{ccc}
\mu & \nu & \rho \\
0 & \mu & 0 \\
0 & 0 & \mu
\end{array}\right)
$$

From the matrix parameters introduce the elements $A, B, C, D \in W$

$$
A:=\lambda \alpha+a \beta+\mu \gamma, B:=\alpha+b \beta+\nu \gamma, C:=c \beta+\rho \gamma, D:=\lambda^{\prime} \alpha+c \beta+\tau \gamma
$$

Take elements $\omega_{i} \in \Lambda^{2} \mathfrak{z}(i=1,2,3)$ and define

$$
\delta\left(e_{i}\right):=D_{\alpha}\left(e_{i}\right) \wedge \alpha+D_{\beta}\left(e_{i}\right) \wedge \beta+D_{\gamma}\left(e_{i}\right) \wedge \gamma+\omega_{i}
$$

In all cases we have

$$
\begin{aligned}
& \delta\left(e_{1}\right)=e_{1} \wedge A+\omega_{1} \\
& \delta\left(e_{2}\right)=e_{2} \wedge A+e_{1} \wedge B+\omega_{2}
\end{aligned}
$$

and $\delta\left(e_{3}\right)$, depending on cases, is equal to

$$
\delta\left(e_{3}\right)=\left\{\begin{array}{l}
e_{3} \wedge D+\omega_{3} \\
e_{3} \wedge A+e_{2} \wedge B+e_{1} \wedge C+\omega_{3} \\
e_{3} \wedge A+e_{1} \wedge C+\omega_{3}
\end{array}\right.
$$


The restriction given by the co-Jacobi identity are

$$
\omega_{1} \wedge A=0=\omega_{2} \wedge A+\omega_{1} \wedge B
$$

and, depending on cases

$$
\begin{array}{ll}
(I) & 0=\omega_{3} \wedge D \\
(I I) & 0=\omega_{3} \wedge A+\omega_{2} \wedge B+\omega_{1} \wedge C \\
(I I I) & 0=\omega_{3} \wedge A+\omega_{1} \wedge C
\end{array}
$$

\section{References}

[BD] A. Belavin, V. Drinfeld, Triangle Equations and Simple Lie Algebras. Mathematical Physics Review, Vol. 4, Soviet Sci. Rev. Sect. C Math. Phys. Rev. 4, Harwood, Chur, Switzerland, (1984), 93-165.

[BS] M. Benayed and E. M. Souidi, Heisenberg Lie Bialgebras as Central Extensions New York J. Math. 4 (1998) 177-183.

[EG] P. Etingof, S. Gelaki, Quasisymmetric and unipotent tensor categories. Math. Res. Lett. 15, No. 5-6, (2008) 857-866.

[FJ] M. Farinati, A.P. Jancsa, Trivial central extensions of Lie bialgebras, Journal of Algebra 390 (2013) 56-76.

[GGI] G. Grantcharov, V. Grantcharov, P. Iliev Solvable Lie algebras and graphs. ArXiv.org/abs/1604.07856

[LW] J. Lauret, C. Will, Einstein solvmanifolds: existence and non-existence questions Mathematische Annalen May 2011, Volume 350, Issue 1, 199-225

[SZ] I. Szymczak S. Zakrzewski, Quantum deformations of the Heisenberg group obtained by geometric quantization Journal of Geometry and Physics, Volume 7, Issue 4, 1990, Pages 553-569. 